\title{
Desarrollo ontogenético y redescripción del adulto de Epidamaeus mitlsensillus (Acari: Oribatida: Damaeidae)
}

\section{Ontogenetic development and redescription of the adult of Epidamaeus mitlsensillus (Acari: Oribatida: Damaeidae)}

\author{
Ricardo Iglesias ${ }^{\bigotimes}$, Rocío Vázquez y José G. Palacios-Vargas \\ Laboratorio de Ecología y Sistemática de Microartrópodos, Departamento de Ecología y Recursos Naturales, Facultad de Ciencias, Universidad \\ Nacional Autónoma de México. Circuito exterior s/n, Ciudad Universitaria, Coyoacán, 04510 México, D. F., México. \\ هiglesias60@yahoo.com
}

\begin{abstract}
Resumen. Se describe e ilustra el desarrollo ontogenético completo de Epidamaeus mitlsensillus Palacios-Vargas, 1984 (larva, protoninfa, deutoninfa y tritoninfa), del volcán Popocatépetl, Estado de México; asimismo, se redescribe el adulto. Los ejemplares fueron aclarados, disecados y montados en preparaciones semipermanentes con líquido de Hoyer para su observación y toma de mediciones. Las ilustraciones, que se presentan en láminas, fueron realizadas con ayuda de cámara clara, entintadas y luego escaneadas.
\end{abstract}

Palabras clave: ontogenia, quetotaxia, Oribatei, morfología, inmaduros.

\begin{abstract}
The complete ontogenetic development of Epidamaeus mitlsensillus Palacios-Vargas, 1984 (larva, protonymph, deutonymph, and tritonymph) from the Popocatepetl Volcano, State of México, is described and illustrated and redescription of the adult is done. The specimens were cleared, dissected and mounted on semi-permanent slides in Hoyer's solution for observation and measurements. Drawings were done using a camera lucida, then inked, scanned, and arranged in plates.
\end{abstract}

Key words: ontogeny, chaetotaxy, Oribatei, morphology, immatures.

\section{Introducción}

Los ácaros oribátidos o criptostigmados (Acarida: Oribatei) forman uno de los grupos de microartrópodos más abundantes en el suelo, hojarasca, humus, musgos, líquenes, nidos, cuevas, así como en troncos y ramas de árboles, vegetación herbácea, e incluso en el entorno peridoméstico (Pérez-Íñigo, 1997). Se conocen en el mundo más de 10000 especies descritas y agrupadas en 1 333 géneros y 181 familias (Schatz, 2004). Sin embargo, el conocimiento de los ácaros oribátidos en México es escaso (aproximadamente 430 taxones) y sólo se tienen algunos inventarios taxonómicos (Palacios-Vargas e Iglesias, 2004, 2007)y varios trabajos de ecología que los incluyen. También se han descrito especies de diversos ambientes (Palacios-Vargas e Iglesias, 1997; Ríos y PalaciosVargas, 1998). La familia Damaeidae Berlese, 1896 está constituida por 13 géneros, 16 subgéneros, 262 especies y 7 subespecies, con distribución cosmopolita excepto en la región antártica (Subías, 2011). Epidamaeus BulanovaZachvatkina, 1957, considerado subgénero de Damaeus

Recibido: 21 marzo 2012; aceptado: 26 junio 2012 por Subías (2004), contiene 77 especies y una subespecie, con distribución holártica, oriental y neotropical (Subías, 2011). Hasta el momento, en México, sólo se han descrito Belba clavasensilla Norton et Palacios-Vargas, 1982 y Epidamaeus mitlsensillus Palacios-Vargas, 1984, ambas del volcán Popocatépetl, Estado de México.

La sistemática de los oribátidos fue establecida por Grandjean (1953) entre otros, basándose tanto en caracteres de los adultos como de los juveniles. El estudio de estos últimos ha adquirido gran importancia, otorgándose cada vez mayor valor a las características de las fases inmaduras, dada su utilidad para establecer relaciones filogenéticas. Por esa razón, en este trabajo se describen todas la fases inmaduras de E. mitlsensillus, especie de la que sólo se había descrito el adulto (Palacios-Vargas, 1984).

\section{Materiales y métodos}

Todos los ejemplares, tanto juveniles como adultos de E. mitlsensillus, fueron recolectados en el volcán Popocatépetl, México, durante los años 1981 a 1983, en la estación II (biotopo suelo) a $3500 \mathrm{~m}$ de altitud por PalaciosVargas (1984). Se hicieron preparaciones en líquido de 
Hoyer de 4 larvas, 7 protoninfas, 3 deutoninfas, 4 tritoninfas y 4 adultos. De estos últimos se hicieron disecciones para poder estudiar las patas. La descripción y los dibujos se realizaron con la ayuda de un microscopio de contraste de fases, marca Carl ZEISS, con cámara clara. Las medidas se presentan en micrómetros $(\mu \mathrm{m})$. La quetotaxia de las patas se da en el siguiente orden: trocánter, fémur, genua, tibia y tarso. La terminología morfológica utilizada fue desarrollada por Grandjean (véase Travé y Vachon, 1975).

\section{Descripción del desarrollo ontogenético}

Epidamaeus mitlsensillus Palacios-Vargas, 1984 (Figs. $1-8)$.

Larva (Figs. 1,2). Cuerpo ovalado, ligeramente redondeado en parte posterior. Longitud total $154 \mu \mathrm{m}(130-192 \mu \mathrm{m})$ $(\mathrm{n}=4)$, anchura $80 \mu \mathrm{m}(63-94 \mu \mathrm{m})$; cuerpo débilmente esclerosado, cutícula blanquecina. Patas y cuerpo finamente cubiertos con delgadas capas de cerotegumento granular, de color grisáceo y tamaño aproximado de un micrómetro. En las patas, estos gránulos se presentan en menor número y tamaño. Prodorso ovalado, con rostro redondeado y sedas de color café claro. A excepción de las sedas ex, todas las del prodorso, incluyendo el sensilo, débilmente recubiertas con cerotegumento de apariencia granular. Sedas rostral $(r o, 80 \mu \mathrm{m})$ y lamelar $(l e ~ 80 \mu \mathrm{m})$ barbuladas y setiformes. Seda lamelar más delgada que la rostral y ambas dirigidas hacia adelante. Sensilo (ss, 273 $\mu \mathrm{m})$ liso, con cerotegumento a partir de la mitad hacia la parte distal, terminando en forma de flagelo. Botridio en forma de copa y bien desarrollado. Sedas interlamelar (in, $115 \mu \mathrm{m})$ y exobotridial $(e x, 48 \mu \mathrm{m})$ localizadas cerca del botridio (cuadro 1). Notogáster también con capa débil de cerotegumento. Porta 12 pares de sedas ligeramente barbuladas, de color café oscuro. Sedas $d a$ y $l p$ son las de mayor longitud, mientras que $c 3, h 2$ y $h 3$ son las más cortas, con terminación en punta y más delgadas; $l p$ ligeramente más gruesa y más larga que $d p$; seda $d a$ barbulada, similar a $d p$ y $l p$. Sedas $l a, l m$ y $c 3$ setiformes y lisas. Las $c 3$ y $l m$ no se insertan sobre un tubérculo, mientras que las $d p$ lo hacen sobre esclerito común. Dentro de la región ventral la placa anal carece de sedas; lateralmente a ésta se observan las glándulas opistosomales y sus aberturas. Fórmula epimeral 2-1-2 (Fig. 2). Todos los estadios ninfales son semejantes morfológicamente a la larva.

Protoninfa (Figs. 3, 4). Longitud del cuerpo $(\mathrm{n}=7) 207$ $\mu \mathrm{m}(184-226 \mu \mathrm{m})$, anchura $94 \mu \mathrm{m}(86-106 \mu \mathrm{m})$; seda ro $153 \mu \mathrm{m}$, seda le $134 \mu \mathrm{m}$, ex $53 \mu \mathrm{m}$, ss $373 \mu \mathrm{m}$. Seda interlamelar sufre reducción en la longitud, con respecto a la larva, $45 \mu \mathrm{m}$. Serie $d(d a, d m$ y $d p$ ) desaparece en ninfas. Notogáster porta 12 pares de sedas. Longitud de éstas visiblemente mayor que en la larva. Todas las sedas notogastrales barbuladas, y parte distal de sedas $c 1, c 2, l a$, $l m, l p$, y $h 3$ es curva o sinuosa y con frecuencia llegan a romperse; sedas $c 1$ dirigidas anteriormente; en protoninfa aparecen sedas $p 1, p 2$ y $p 3$ (Figs. 3 y 4). Sedas más largas son las $h 1, c 1$ y $c 2$; las más cortas son $p 2, p 3$ y $c 3 ; h 2$, $p 1, p 2$ y $p 3$ delgadas y ligeramente barbuladas. Tubérculos de sedas $c 1$ presentan coloración café claro y comparten un esclerito común. Presencia de cornículo acronótico $k$, alargado, liso, adelgazándose al final. Con la exuvia de la larva. Por el lado ventral, placa genital con par de sedas y debajo de ellas par de papilas. Placa anal carece de sedas. Fórmula epimeral 3-1-2-1, con sedas laterales ligeramente barbuladas. Se observa un grado de esclerotización ligeramente mayor al de la larva; lirifisura ips localizada arriba de la seda $p 3$, ip en medio de $h 2$ y $h 3$. Lirifisura ih y abertura de la glándula opistosomal ( $\mathrm{gla}$ ) localizadas lateralmente a sedas $p 2$ y $p 3$ (Figs. 3 y 4). Gnatosoma: infracapítulo y quelíceros sólo cambian de tamaño en el desarrollo de las etapas subsecuentes.

Deutoninfa (Figs. 5, 6). Longitud del cuerpo $(\mathrm{n}=3) 480$ $\mu \mathrm{m}(469-501 \mu \mathrm{m})$; anchura $241 \mu \mathrm{m}(109-294 \mu \mathrm{m})$. Región del prodorso subtriangular con las ro y le ligeramente barbuladas. Se observa también par de carinas laterales que parten del nivel de seda lamelar hacia la base del botridio. Longitud de sedas rostral, lamelar, exobotridial así como el sensilo (ss) presentan aumento de tamaño con respecto a protoninfa (Cuadro 1). Notogáster con 12 pares de sedas de diferente tamaño con marcada barbulación y parte distal muy delgada. Sedas $c 1, c 2, c 3, l a, h 1$ sufren reducción con respecto a la protoninfa. Seda $c 3$ sigue siendo la más corta. Distribución de las mismas sin cambios. En parte posterior se presenta cornículo acronótico, largo y trunco en parte distal. Se observan, asimismo, sobre el notogáster, 2 exuvias provenientes de la larva y la protoninfa. En región ventral, 3 pares de sedas adanales (ad1, ad2, ad3) setiformes, delgadas y ligeramente barbuladas; un par de lirifisuras iad y un par de sedas adgenitales $(a g)$. Lirifisuras ips localizadas por arriba de las sedas $p 2$ y $p 3$. Placa genital con 3 pares de sedas, y por debajo 2 pares de papilas genitales. Fórmula epimeral 3-1-2-2.

Cuadro 1. Longitud de las sedas prodorsales en micrómetros $(\mu \mathrm{m})$

\begin{tabular}{lccccc}
\hline & ro & Le & ss & In & Ex \\
\hline LV & 80 & 80 & 273 & 115 & 48 \\
PN & 153 & 134 & 373 & 45 & 53 \\
DN & 170 & 198 & 375 & 68 & 78 \\
TN & 225 & 257 & 362 & 82 & 97 \\
AD & 92 & 112 & 133 & 82 & 35 \\
\hline
\end{tabular}




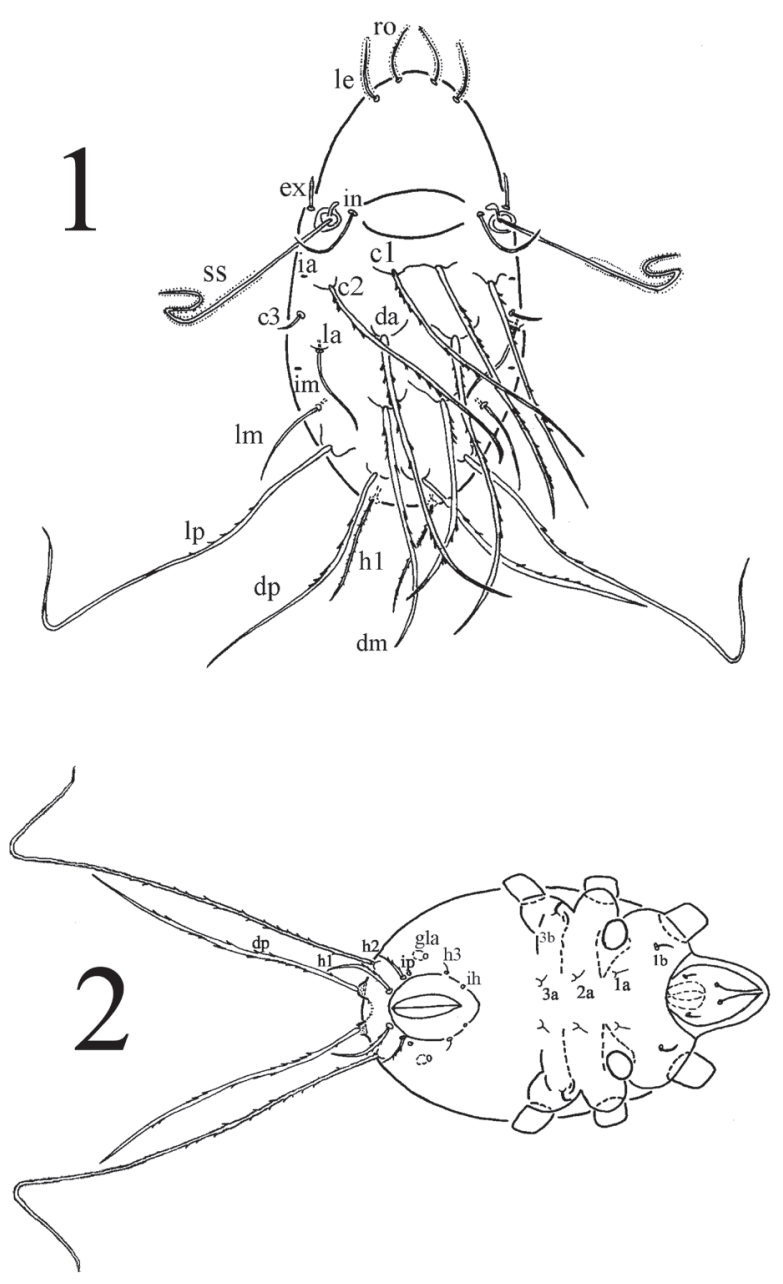

Figuras 1-2. Larva de Epidamaeus mitlsensillus; 1, vista dorsal; 2 , vista ventral.

Tritoninfa (Figs. 7, 8). Longitud del cuerpo $(\mathrm{n}=4) 613 \mu \mathrm{m}$ (576-632 $\mu \mathrm{m})$, anchura $302 \mu \mathrm{m}(235-383 \mu \mathrm{m})$. Longitud de sedas rostral, lamelar, interlamelar y exobotridial aumenta de tamaño en relación con las de la deutoninfa. Sin embargo, el sensilo sufre reducción en longitud y forma, volviéndose corto y setiforme; carinas laterales del prodorso más evidentes en esta etapa. Botridio conserva forma cónica. Sedas rostrales y lamelares dirigidas hacia adelante; $c 3$ barbulada y pequeña (Cuadro 2). Forma del cuerpo ovalada con región posterior más angosta. Región del notogáster porta 3 exuvias (larva, protoninfa y deutoninfa) conectadas una con otra por medio del cornículo acronótico $k$, y 12 pares de sedas insertadas sobre apófisis y ligeramente más gruesas y barbuladas que en la deutoninfa. Tubérculos de sedas cl se encuentran sobre esclerito color café claro. Sedas
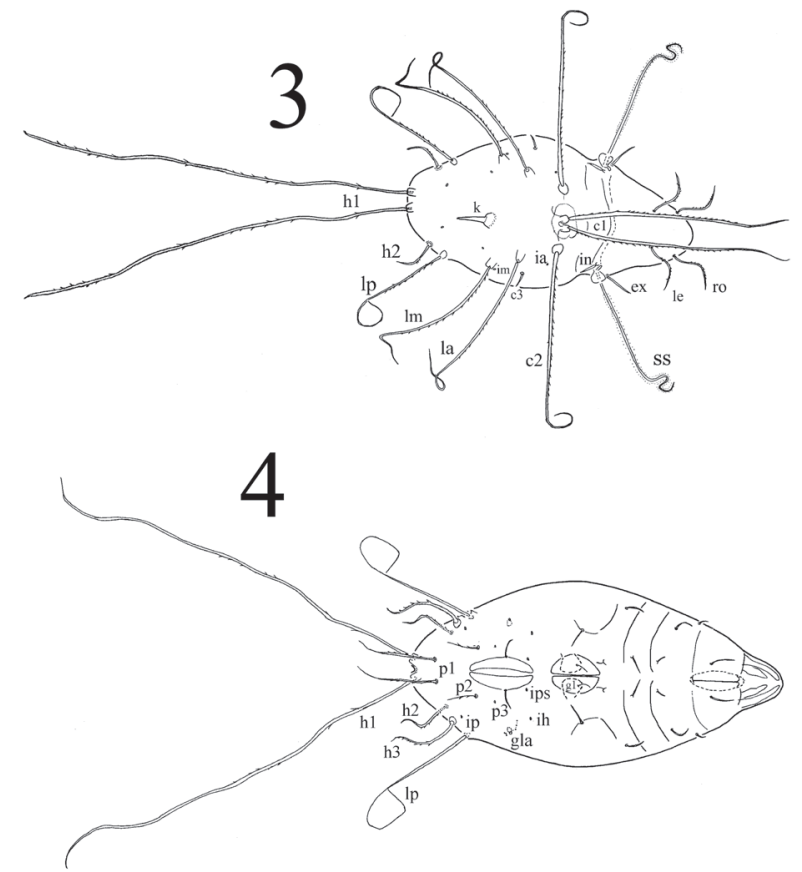

Figuras 3-4. Protoninfa de E. mitlsensillus; 3, vista dorsal; 4, vista ventral.

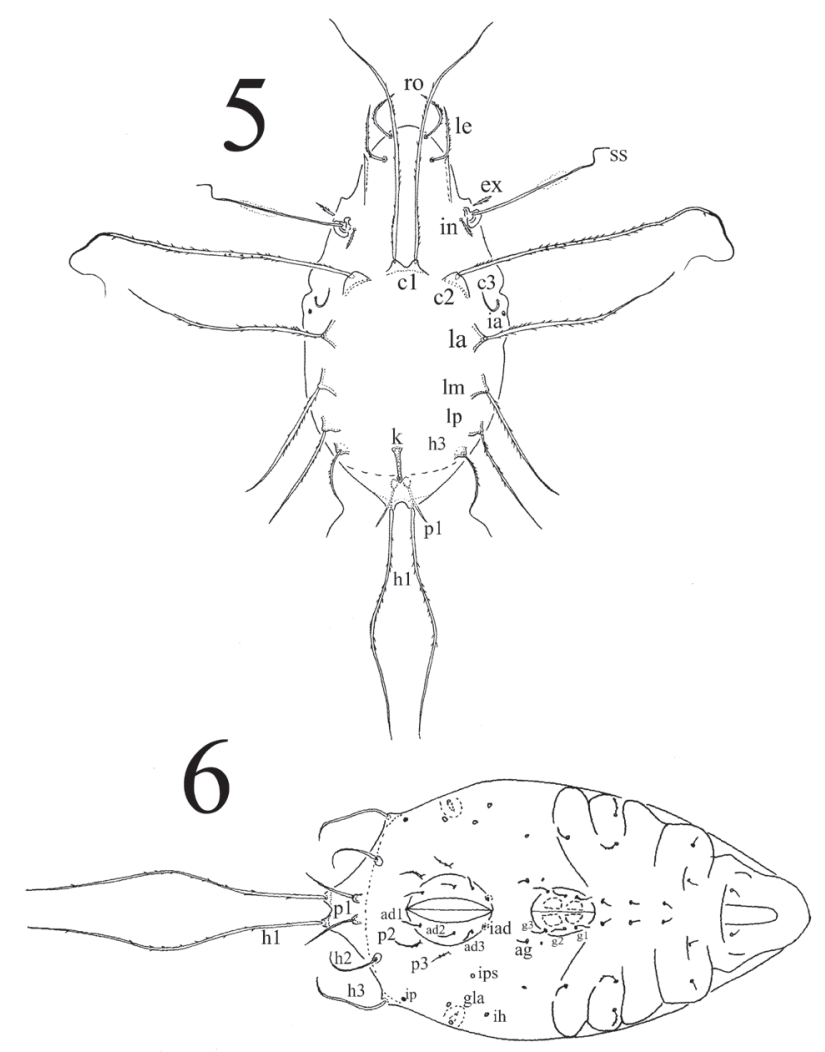

Figuras 5-6. Deutoninfa de E. mitlsensillus; 5, vista dorsal; 6, vista ventral. 

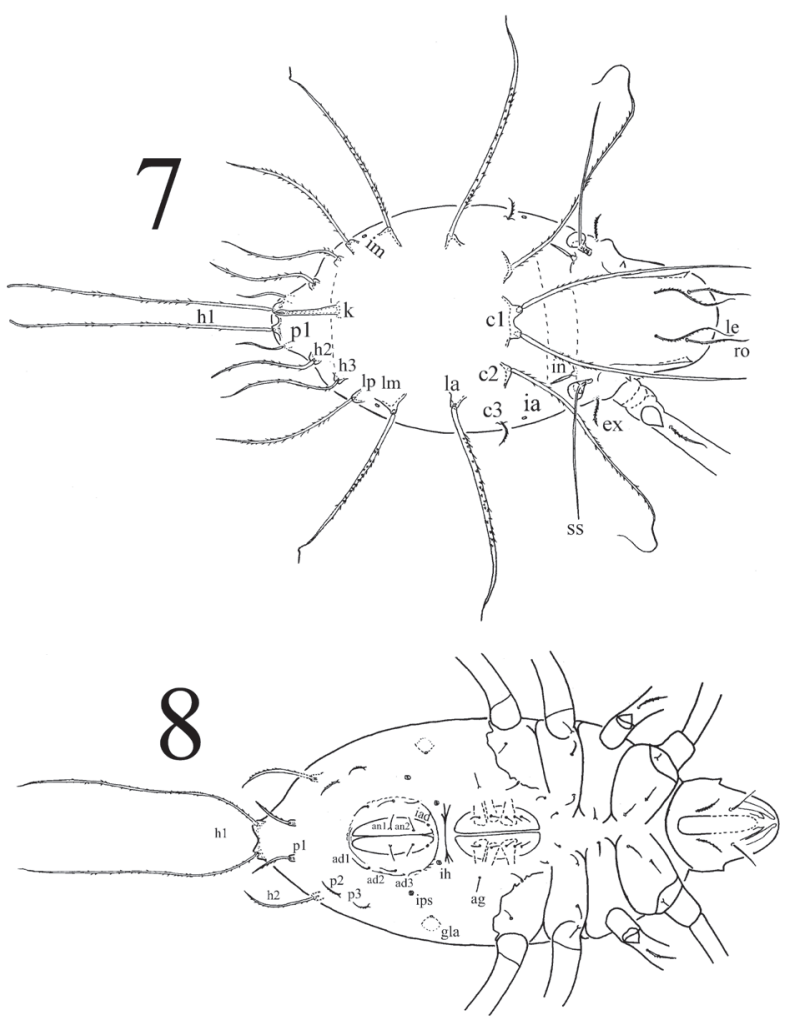

Figuras 7-8. Tritoninfa de E. mitlsensillus; 7, vista dorsal; 8, vista ventral.

$l a, l m$ y $l p$ setiformes y barbuladas. Placa anal con 2 pares de sedas (an1 y an2) y hay 3 pares de adanales (ad1, ad2, ad3). Placa genital con 5 pares de sedas finas y conspicuas. Lateralmente se observa par de sedas adgenitales. En región epimérica se observan apodemas definidos; fórmula epimeral 3-1-3-3. Sólo un ejemplar presentó asimetría en el epímero 3 con una seda de más. Gnatosoma con infracapítulo, pedipalpos, y quelíceros característicos de la familia. Patas: en el Cuadro 3 se presenta la quetotaxia de las patas indicando cuáles sedas son las que aparecen en cada etapa del desarrollo, así como las que se pierden (perd.). En el Cuadro 4 se presentan las dimensiones de los artejos.
Adulto. Redescripción (Figs. 9-15). Longitud del cuerpo $(\mathrm{n}=4), 745 \mu \mathrm{m}(687-766 \mu \mathrm{m})$; anchura del notogáster $457 \mu \mathrm{m}(408-489 \mu \mathrm{m})$. Cerotegumento: Cuerpo y patas cubiertos por delgada capa de cerotegumento reticular, algunas veces cónico, surcos en la región dorsosejugal y ventrosejugal, con tubérculos en la región laterosejugal más alargados y filamentosos. Sólo porción distal del tarso de las patas, rostro, gnatosoma y sedas libres de cerotegumento. Predorso subtriangular, integumento liso, con 2 pares de enantiófisis dorsosejugales ( $D a$ y $B 1)$. Seda lamelar (le) finamente barbulada; rostral (ro) más delgada con barbulaciones cortas y finas. Enantiófisis postbotridial poco desarrollada. Sensilo (ss) setiforme con punta en forma de flecha, y longitud de $133 \mu \mathrm{m}$; sedas exobotridiales (ex) pequeñas, finamente barbuladas $\mathrm{y}$ ocultas en vista dorsal por el botridio. Seda interlamelar (in) mide $82 \mu \mathrm{m}$ sin barbulaciones (Fig. 9). Notogáster (Fig. 9) ligeramente elíptico en vista perpendicular con sutura circumgástrica; aproximadamente 1.2 veces más largo que ancho. Espina adnata $(s a)$ pequeña y aguda. Con 11 pares de sedas de color café oscuro, lisas y ligeramente curvas, radialmente dirigidas, disminuyendo gradualmente en grosor. Sedas $p s 1, p s 2$ y $p s 3$, laterales y reducidas, hacia la parte distal, con terminación curva, más o menos paralelas a la sutura circumgástrica. Las sedas $c 1$ y $c 2$ dirigidas hacia la parte anterior. Las demás en dirección posterior, excepto las sedas $p s$. Sedas aumentan en longitud desde la seda $c 1$ hasta la $l m$; de la $p 1$ a la $p 3$ disminuyen en longitud. Glándulas opistosomales y lirifisuras propias de la familia. Adultos portan las exuvias ninfales. Ausencia de sedas $c 3$. Región ventral (Fig.10): enantiófisis esternal $E 2 a$ difícil de observar y $E 2 p$ no está bien desarrollada, sino representada sólo por cresta gruesa a cada lado del segundo surco epimeral. Tubérculo Va bien desarrollado, subtriangular, con punta redondeada. $V p$ pequeño y difícil de ver. Tubérculo $s a$ alargado con terminación aguda; $s p$ bien desarrollado en posición opuesta a $s a$ formando una enantiófisis. Fórmula epimeral 3-1-3-4 con sedas lisas; sólo sedas $3 c$ y $4 c$ epimerales flageladas. Placas genitales con 6 pares de sedas; lateralmente un par de sedas adgenitales. Con 3 pares de papilas genitales. Seda ad3 desplazada lateralmente. Discidium (di) pobremente

Cuadro 2. Longitud de las sedas notogastrales en micrómetros $(\mu \mathrm{m})$

\begin{tabular}{|c|c|c|c|c|c|c|c|c|c|c|c|c|c|c|c|}
\hline & $c_{1}$ & $c_{2}$ & $c_{3}$ & $d a$ & $d m$ & $d p$ & la & $\operatorname{lm}$ & $l p$ & $h_{1}$ & $h_{2}$ & $H_{3}$ & $p_{1}$ & $P_{2}$ & $p_{3}$ \\
\hline LV & 375 & 333 & 60 & 380 & 325 & 338 & 112 & 100 & 445 & 135 & 68 & 45 & - & - & - \\
\hline PN & 578 & 585 & 48 & - & - & - & 387 & 332 & 277 & 720 & 105 & 135 & 102 & 57 & 23 \\
\hline $\mathrm{DN}$ & 275 & 188 & 24 & - & - & - & 192 & 450 & 375 & 312 & 155 & 175 & 118 & 75 & 55 \\
\hline $\mathrm{TN}$ & 683 & 608 & 58 & - & - & - & 663 & 593 & 695 & 888 & 235 & 340 & 133 & 70 & 55 \\
\hline $\mathrm{AD}$ & 107 & 127 & - & - & - & - & 135 & 155 & 145 & 75 & 110 & 75 & 45 & 37 & 35 \\
\hline
\end{tabular}


Cuadro 3. Ontogenia quetotáxica de las patas de Epidamaeus mitlsensillus*

\begin{tabular}{|c|c|c|c|c|c|}
\hline & Trocánter & Fémur & Genua & Tibia & Tarso \\
\hline \multicolumn{6}{|l|}{ Pata I } \\
\hline Larva & - & bv", d & d $\sigma l ' l^{\prime \prime}$ & d $\varphi l ' l ’ v '$ & $(\mathrm{ft})(\mathrm{tc})(\mathrm{p})(\mathrm{u})(\mathrm{a}) \mathrm{s}(\mathrm{pv})(\mathrm{pl})$ e $\omega 1$ \\
\hline Protoninfa & - & - & - & - & $\omega 2$ \\
\hline Deutoninfa & v' & (1) & v' & v" $\varphi 2$ & - \\
\hline Tritoninfa & - & $\mathrm{v} 1 ”$ & - & - & (it) \\
\hline Adulto & - & v1'v2"' & - & d perd. & (v) \\
\hline \multicolumn{6}{|l|}{ Pata II } \\
\hline Larva & - & bv", d & d $\sigma l^{\prime} l^{\prime \prime}$ & d $\varphi l ' l ' v '$ & $(\mathrm{ft})(\mathrm{tc})(\mathrm{p})(\mathrm{u})(\mathrm{a}) \mathrm{s}(\mathrm{pv}) \omega 1$ \\
\hline Protoninfa & - & - & - & - & - \\
\hline Deutoninfa & v' & (1) & v' & $1 ”$ & $\omega 2$ \\
\hline Tritoninfa & - & - & - & v" & (it) \\
\hline Adulto & - & (v) & - & - & (v) \\
\hline \multicolumn{6}{|l|}{ Pata III } \\
\hline Larva & - & ev', d & dol' & $d \varphi l ' v '$ & $(\mathrm{ft})(\mathrm{tc})(\mathrm{p})(\mathrm{u})(\mathrm{a}) \mathrm{s}(\mathrm{PV})$ \\
\hline Protoninfa & v' & - & - & - & - \\
\hline Deutoninfa & 1 ' & 1 ' & v' & v' & - \\
\hline Tritoninfa & - & - & - & - & (it) \\
\hline Adulto & - & v' & - & - & (v) \\
\hline \multicolumn{6}{|l|}{ Pata IV } \\
\hline \multicolumn{6}{|l|}{ Larva } \\
\hline Protoninfa & - & - & - & - & $\mathrm{ft}^{\prime \prime}(\mathrm{p})(\mathrm{u})(\mathrm{pv})$ \\
\hline Deutoninfa & v' & ev', d & $\mathrm{d}, \mathrm{l}$, & $d \varphi l ' v '$ & (tc) (a) $\mathrm{s}$ \\
\hline Tritoninfa & - & 1 ' & v' & v" & - \\
\hline Adulto & - & $\mathrm{v}$ & - & v perd. & (v) \\
\hline
\end{tabular}

*Sedas que aparecen en cada estadio; los paréntesis indican que aparecen juntas.

perd. $=$ se pierde

Cuadro 4. Longitud promedio de patas y artejos I, II, III y IV en micrómetros

\begin{tabular}{lcccccc}
\hline & Trocánter & Fémur & Genua & Tibia & Tarso & Longitud total \\
\hline Pata I & 44 & 153 & 55 & 79 & 166 & 497 \\
Pata II & 32 & 119 & 48 & 63 & 763 & 405 \\
Pata III & 102 & 101 & 44 & 119 & 41 & 214 \\
Pata IV & 128 & 145 & 70 & 674 & 676 \\
\hline
\end{tabular}

desarrollaado. Gnatosoma: Mentum del infracapítulo cuadrado. Con microtubérculos en la región lateral. Quelíceros y pedipalpos típicos de la familia. Patas (Figs. 12-15) con áreas porosas, ligeramente desarrolladas en fémur, tibia y tarso. Trocánter III con áreas porosas; pata II la más pequeña y IV la más larga de todas (Cuadro 4). Quetotaxia de patas del trocánter al tarso (fámulus incluido, número de solenidios entre paréntesis) como sigue: pata I, 1-7-4(1)-4(2)-20(2); pata II, 1-6-4(1)-4(1)-17(2); pata III, 2-4-3(1)3(1)-17; pata IV, 1-4-3-3(1)-14. No se observan variaciones (Cuadro 3). La forma y distribución de las sedas de las patas se muestran en las Figs. 12-15.

La larva de E. mitlsensillus es similar a la de $E$. kamaensis Ermilov y Lochynska, 2009 en la forma del cuerpo y la longitud de las sedas notogastrales, las cuales se encuentran insertadas sobre apófisis. Difieren en que la primera especie tiene las sedas ro y le cubiertas de cerotegumento, el sensilo es largo, fino y con la parte distal en forma de flagelo. En la protoninfa de E. mitlsensillus el sensilo es largo y con la parte distal flagelar; en E. kamaensis es setiforme y barbulado; además la ex es casi 3 veces más grande que la in, mientras que en E. mitlsensillus la misma seda es apenas 1.5 veces más grande. En la última etapa ninfal, la tritoninfa, los sensilos de ambas especies son largos y setiformes pero en la especie en estudio son lisos y algo más cortos mientras que en E. kamaensis son barbulados (Ermilov y Lochynska, 2009). 


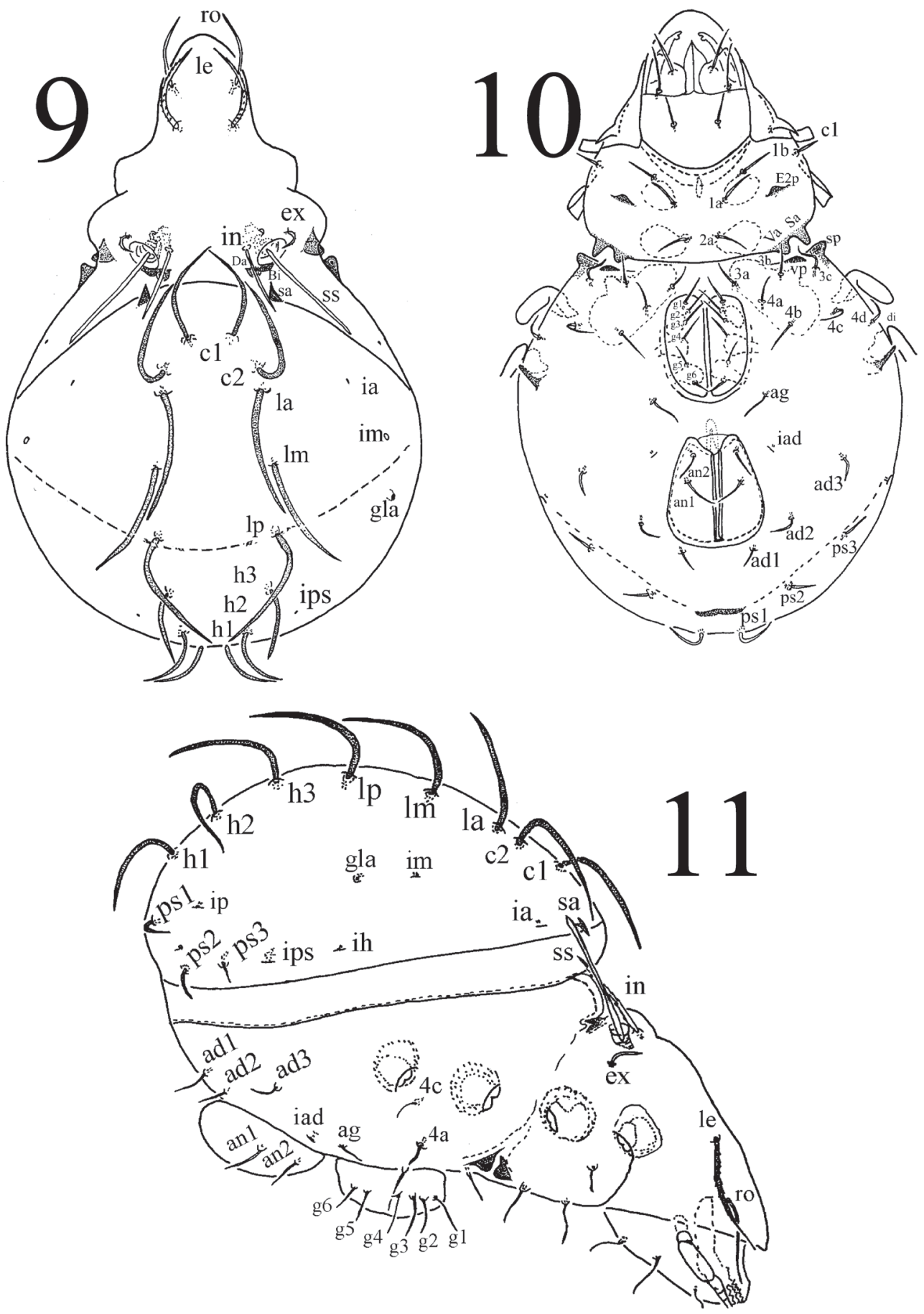

Figuras 9-11. Adulto de Epidamaeus mitlsensillus; 9, vista dorsal; 10, vista ventral; 11, vista lateral. 

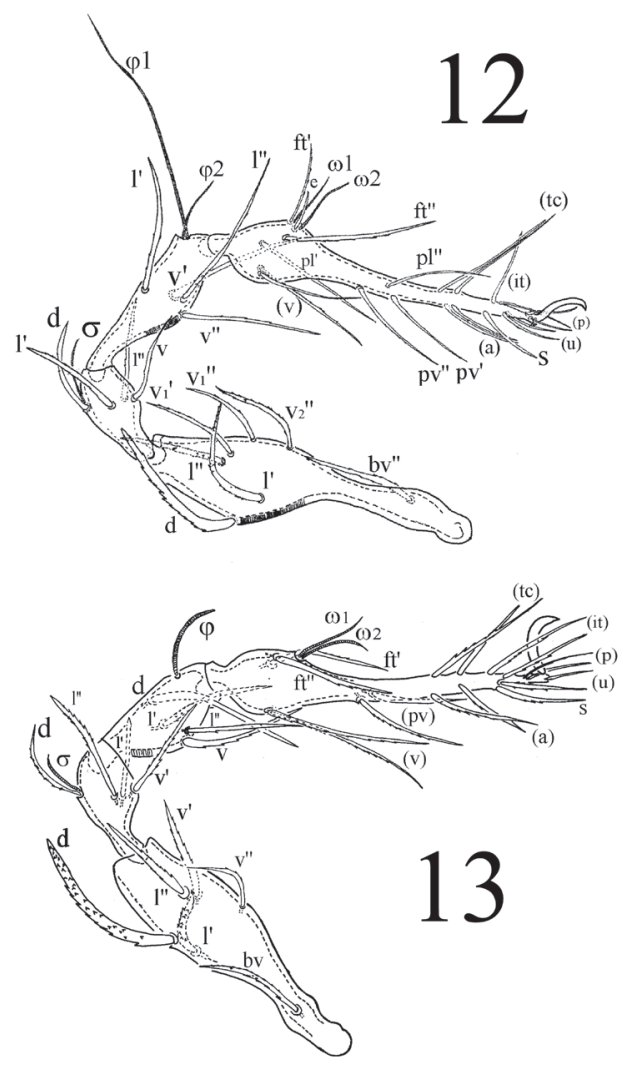

Figuras 12-13. Patas de E. mitlsensillus; 12, pata I de fémur a tarso; 13, pata I de fémur a tarso.

\section{Comentarios taxonómicos}

De las 2 especies de Damaeidae que se conocen de México, sólo las ninfas y el adulto de E. mitlsensillus conservan las exuvias de la larva y estados precedentes, es así que la tritoninfa lleva encima de ella (sujetas por el cornículo acronótico, $k$ ) las mudas de la larva, protoninfa y deutoninfa. El adulto lleva todas ellas incluyendo la de la tritoninfa. Contrario a lo que ocurre en Belba clavasensilla, que no conserva las exuvias. Por lo que $E$. mitlsensillus sigue el patrón de desarrollo más común de la familia.

Las sedas ro y le de E. mitlsensillus son relativamente cortas, y están muy separadas entre ellas en todas las etapas de desarrollo. La seda in es larga en la larva, corta en las ninfas y en el adulto relativamente larga, mientras que la seda $e x$ es pequeña en todas las etapas del desarrollo. El botridio siempre es grande, redondeado y en forma de embudo. El sensilo es relativamente largo en la larva $(273 \mu \mathrm{m})$, medianamente largo en la protoninfa $(373 \mu \mathrm{m})$ y deutoninfa $(375 \mu \mathrm{m})$, corto en la tritoninfa $(362 \mu \mathrm{m})$ y mucho más corto en el adulto $(133 \mu \mathrm{m})$, su ápice es acuminado y ondulado en las etapas ninfales, volviéndose grueso y en forma de punta de flecha en el adulto (Cuadro 1).
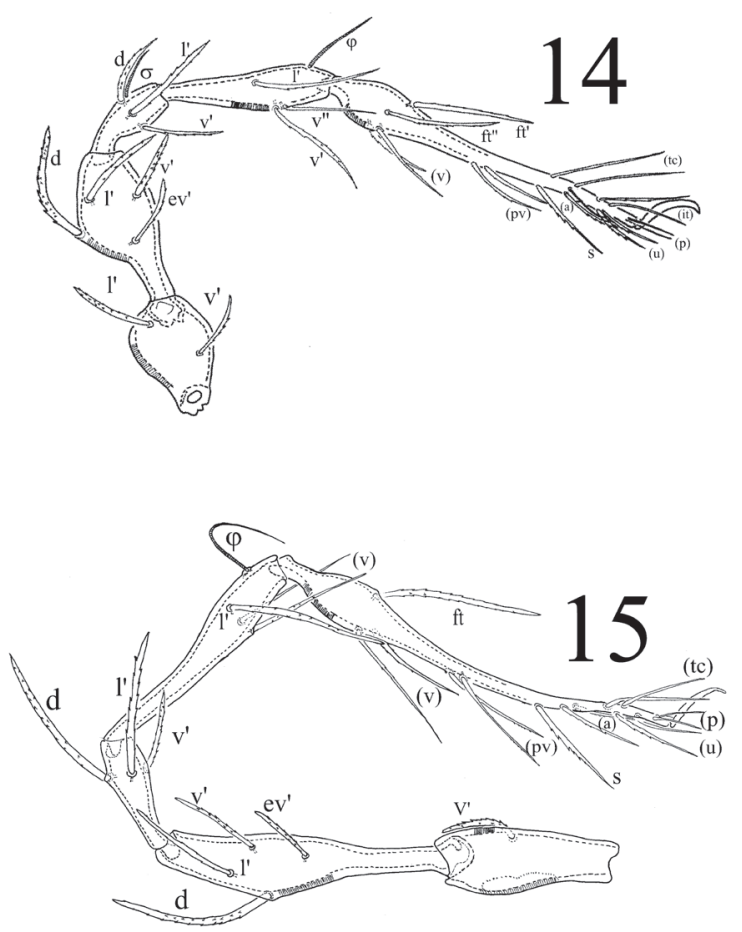

Figuras 14-15. Patas de E. mitlsensillus; 14, pata III de trocánter a tarso; 15, pata IV de trocánter a tarso, en vista antiaxial.

En el curso del desarrollo ontogenético de Epidamaeus se denota una reducción de la quetotaxia del notogaster (de 12 pares en los juveniles a 11 en los adultos), mientras que en las patas ocurre lo contrario, paulatinamente van incrementándose las sedas, lo que parece ser común en el género, pero no en la familia, de acuerdo con el reciente trabajo de Seniczak y Seniczak (2011). Las sedas coxiesternales también tienen un desarrollo normal como en los miembros de la familia. El cornículo acronótico se encuentra entre las sedas $l m$ en la protoninfa y entre las sedas $h 3$ en la deutoninfa y la tritoninfa; su tamaño varía de $24 \mu \mathrm{m}$ en la protoninfa a $75 \mu \mathrm{m}$ en la tritoninfa, además primero es cónico y corto, volviéndose alargado y truncado. Al parecer es un carácter autopomórfico, ya que su posición varía tanto en el nivel genérico como en el específico, según Seniczak y Seniczak (2011), y su uso puede ser de utilidad en análisis filogenéticos. Al comparar los caracteres entre las diferentes especies del género se notó que existe una estabilidad en muchos de ellos y que los pocos caracteres que permiten hacer las diagnosis específicas y servirán para un futuro análisis de las relaciones filogenéticas son el número de sedas del trocánter III, así como las de los tarsos I, II y IV, la forma del sensilo, la longitud de las sedas de grupo $p s$, la forma de las espinas adnatas y las enantiófisis, así como la 
barbulación y longitud de las sedas ventrales (epimerales, genitales y anales).

El desarrollo ontogenético de E. mitlsensillus en la quetotaxia de las patas es similar a E. kamaensis ya que no se encontraron diferencias evidentes, por lo que parece ser una quetotaxia constante en el género. Las características de los estados inmaduros, como el tamaño y la forma de las sedas notogastrales e igualmente el sensilo, cambian de manera gradual desde la larva hasta la tritoninfa. También se presentan otros caracteres de la especie, como las apófisis prodorsales y ventrales y las espinas adnatas, que en las etapas ninfales no existen.

En las nuevas observaciones del adulto de $E$. mitlsensillus se detectó una diferencia en la forma del discidium con respecto a la descrita por Palacios-Vargas (1984), quien lo describió como romo; sin embargo, se notó que es de tipo cónico y alargado.

\section{Agradecimientos}

A Roy A. Norton, de la Universidad de Nueva York, Siracusa, por la valiosa información bibliográfica proporcionada. A Jorge Paniagua Nucamendi, por su gran apoyo en los trabajos de digitalización y edición de imagen.

\section{Literatura citada}

Ermilov, S. G. y M. Lochynska. 2009. Morphology of juvenile stages of Epidamaeus kamaensis (Sellnick, 1925) and Porobelba spinosa (Sellnick, 1920) (Acari: Oribatida: Damaeidae). Annales Zoologici 59:527-544.

Grandjean, F. 1953. Essai de classification des Oribates (Acariens). Bulletin de la Société Zoologique de France 78:421-446.
Palacios-Vargas J. G. 1984. A new Mexican Epidamaeus (Oribatei: Damaeidae). Entomological News 95:23-26.

Palacios-Vargas. J. G. and R. Iglesias. 1997. A new species of Malaconothrus Berlese from Yucatán, México (Acari: Oribatida: Malaconothridae). Genus 8:109-113.

Palacios-Vargas, J. G. y R. Iglesias. 2004. Oribatei (Acari). In Biodiversidad, taxonomía y biogeografía de artrópodos: Hacia una síntesis de su conocimiento, vol. IV. J. LlorenteBousquets, J. J. Morrone, O. Yánez e I. Vargas (eds.). Facultad de Ciencias, Universidad Nacional Autónoma de México, México, D. F. p. 431-468.

Palacios-Vargas, J. G. y R. Iglesias. 2007. Ácaros oribátidos. In Biodiversidad de la Faja Volcánica Transmexicana. I. Luna, J. J. Morrone y D. Espinosa (eds.). Las Prensas de Ciencias, Facultad de Ciencias, UNAM. México, D. F. p. 345-356.

Pérez-Íñigo, C. 1997. Acari, Oribatei, Gymnonota I. In Fauna Ibérica, vol. 9, M. A. Ramos et al. (eds.). Museo Nacional de Ciencias Naturales, CSIC. Madrid. 374 p.

Ríos, G. y J. G. Palacios-Vargas. 1998. Especies nuevas de Scapheremaeus (Acari: Oribatei: Cymbaeremaeidae) de México. Anales del Instituto de Biología, Universidad Nacional Autónoma de México, Serie Zoología 69:181-215.

Schatz, H. 2004. Diversity and global distribution of Oribatid mites (Acari: Oribatida): evaluation of the present state of knowledge. Phytophaga 14:485-500.

Seniczak, A. y A. Seniczak. 2011. Differentiation of external morphology of Damaeidae (Acari: Oribatida) in light of the ontogeny of three species. Zootaxa 2775:1-36.

Subías, L. S. 2011. Listado sistemático, sinonímico y biogeográfico de los ácaros oribátidos (Acariformes: Oribatida) del Mundo (Excepto fósiles). http://www.ucm.es/info/zoo/Artropodos/ Catalogo.pdf; última consulta: 15.VI.2012.

Travé, J. y M. Vachon. 1975. François Grandjean, 1882-1975. (Notice biographique et bibliographique). Acarologia 17:1-19. 